\title{
Duration-dependence of the effect of treadmill exercise on hyperactivity in attention deficit hyperactivity disorder rats
}

\author{
Eun-Sang Ji', Chang-Ju Kim', Jun Heon Park2,3, Geon Ho Bahn",* \\ 'Department of Physiology, College of Medicine, Kyung Hee University, Seoul, Korea \\ ${ }^{2}$ Kohwang Medical Research Institute, College of Medicine, Kyung Hee University, Seoul, Korea \\ ${ }^{3}$ Department of Psychiatry, College of Medicine, Kyung Hee University, Seoul, Korea
}

Attention-deficit hyperactivity disorder (ADHD) is a common neurobehavioral disorder, and its symptoms are hyperactivity and deficits in learning and memory. Physical exercise increases dopamine synthesis and neuronal activity in various brain regions. In the present study, we investigate the duration-dependence of the treadmill exercise on hyperactivity in relation with dopamine expression in ADHD. Spontaneously hypertensive rats were used for the ADHD rats and Wistar-Kyoto rats were used for the control rats. The rats in the exercise groups were forced to run on a treadmill for $10 \mathrm{~min}, 30 \mathrm{~min}$, and 60 min once daily for 28 consecutive days. For this experiment, open field test and immunohistochemistry for tyrosine hydroxylase were conducted. The present results revealed that ADHD rats showed hyperactivity, and tyrosine hy- droxylase expression in the striatum and substantia nigra were decreased in ADHD rats. Treadmill exercise alleviated hyperactivity and also increased TH expression in ADHD rats. Treadmill exercise for 30 min per day showed most potent suppressing effect on hyperactivity, and this dose of treadmill exercise also most potently inhibited tyrosine hydroxylase expression. The present study suggests that treadmill exercise for 30 min once a day is the most effective therapeutic intervention for ADHD patients.

Keywords: Attention-deficit hyperactivity disorder, Treadmill exercise, Duration-dependence, Hyperactivity, Dopamine

\section{INTRODUCTION}

Attention deficit/hyperactivity disorder (ADHD) is a neurodevelopmental disorder and this disorder affects about $8-12 \%$ of children at school age (6-12 yr) (Pastor and Reuben 2008). ADHD is characterized by three major behavioral symptoms: inattention, hyperactivity, and impulsivity. In addition, ADHD patients often exhibit learning difficulty and cognitive impulsiveness (Wilens and Dodson, 2004). Although its etiology is unknown, the most likely mechanism for ADHD is damage to the dopaminergic and noradrenergic pathway (Biederman and Spencer, 1999; Sagvolden and Sergeant, 1998).

It has been widely suggested that dopamine plays an important role for the modulation of neuroendocrine functions, cognition, at- tention, reward, and behaviors including motor activity. Dysfunction of dopamine signaling is related to the brain disorders such as Parkinson's disease and ADHD (Volkow et al., 2007; Yoon et al., 2007). Previous studies reported that dysfunction of dopamine signaling in the midbrain substantia nigra $(\mathrm{SN})$ is one of the most possible mechanisms of the behavioral symptoms of ADHD (Bowton et al., 2010). SN plays an important role in brain functions, particularly in movement, motor planning, reward seeking, learning, and addiction. Many functions of $\mathrm{SN}$ are mediated through the striatum. The nigral dopaminergic input to the striatum via the nigrostriatal pathway is intimately linked with the striatum's function (Nicola et al., 2000). Tyrosine hydroxylase (TH) is the enzyme synthesizing catecholamine neurotransmitters, and it converts L-tyrosine to L-dihydroxyphenylalanine (L-DOPA), which is
${ }^{*}$ Corresponding author: Geon Ho Bahn

Department of Psychiatry, College of Medicine, Kyung Hee University,

26 Kyungheedae-ro, Dongdaemun-gu, Seoul 130-701, Korea

Tel: +82-2-958-8556, Fax: +82-2-957-1997, E-mail: mompeian@khu.ac.kr

Received: April 18, 2014 / Accepted: April 21, 2014
This is an Open Access article distributed under the terms of the Creative Commons Attribution Non-Commercial License (http://creativecommons.org/licenses/by-nc/3.0/) which permits unrestricted non-commercial use, distribution, and reproduction in any medium, provided the original work is properly cited. 
the rate-limiting step in the dopamine synthesis. TH activity is progressively decreased according to the loss of dopamine neurons in the SN (Haavik and Toska, 1998). TH immunohistochemistry is a useful technique detecting injury or death of dopaminergic fibers or cell bodies (Hurley et al., 2004; Kim et al., 2011).

In ADHD children, physical movement improved working speed and social behavioral problems, and diminished hyperactivity (Majorek et al., 2004). Kim et al. (2011) reported that treadmill exercise ameliorated symptoms of ADHD rats. Despite a variety of studies on the effect of treadmill exercise on various neuropsychiatric disorders (Heo et al., 2014; Jee et al., 2008; Kim et al., 2014), there is no available data on the duration-dependence of the effects of treadmill exercise on the symptoms relief in the ADHD patients.

In the present study, we investigated the duration-dependence of the effect of treadmill exercise on the hyperactivity in relation with DA expression in the ADHD rats. For this study, open field test and immunohistochemistry for $\mathrm{TH}$ were performed.

\section{MATERIALS AND METHODS}

\section{Experimental animals}

Adult male spontaneously hypertensive rats (SHR) weighing $210 \pm 10 \mathrm{~g}$ were used as the ADHD animal model, because SHR displays the major symptoms of ADHD, such as inattention, hyperactivity, and impulsiveness (Sagvolden, 2000). SHR showing hyperactivity in open field test was selected for this experiment. Meanwhile, Wistar-Kyoto rats (WKYR) weighing 210 $10 \mathrm{~g}$ were used as the control rats in this study, according to the previous study (Sagvolden, 2000). The rats were housed under controlled temperature $\left(20 \pm 2^{\circ} \mathrm{C}\right)$ and lighting conditions (07:0019:00), with food and water made available ad libitum throughout the experiments. The animals were randomly divided into four groups ( $\mathrm{n}=10$ in each group): the control group, the ADHD group, the ADHD and 10 min treadmill exercise group, the $\mathrm{ADHD}$ and 30 min treadmill exercise group, and the ADHD and $60 \mathrm{~min}$ treadmill exercise group. All animal procedures were performed in accordance with the animal care guidelines of the National Institutes of Health (NIH) and the Korean Academy of Medical Sciences.

\section{Treadmill exercise protocol}

The rats in the treadmill exercise groups were subjected to run on a treadmill for $10 \mathrm{~min}, 30 \mathrm{~min}$, and $60 \mathrm{~min}$ once a day, five times a week, continued for 28 days. Exercise load for the exercise groups consisted of running at a speed of 2 meters/min for the first $5 \mathrm{~min}$, at a speed of 5 meters $/ \mathrm{min}$ for the next $5 \mathrm{~min}$, and then at a speed of 8 meters $/ \mathrm{min}$ for the last $20 \mathrm{~min}$, with the $0^{\circ}$ inclination. This intensity corresponded to the low-intensity treadmill exercise (\% maximal oxygen consumption) according to the rats in this age (Bedford et al., 1979).

\section{Open field test}

Activity was determined using the open field test. The animals were randomly assigned to an order of testing and placed in a white square open field arena $(100 \mathrm{~cm} \times 100 \mathrm{~cm})$ made of wood. As the previously reported method (Durand et al., 1999), it was enclosed with 40-cm high walls and placed under strong illumination (200 lux). The arena was divided into 25 squares (each square is $20 \times 20 \mathrm{~cm}$ ), defined as 9 central and 16 peripheral squares. The animal was placed in the center of the arena and left free to explore the environment for $1 \mathrm{~min}$. After that time, the numbers of squares that the rat crossed were recorded for $5 \mathrm{~min}$.

\section{Tissue preparation}

The rats were sacrificed 29 days after the starting of the experiment. The animals were fully anesthetized using Zoletil $50^{\circledR}(10$ $\mathrm{mg} / \mathrm{kg}$, i.p.; Vibac Laboratories, Carros, France). The anesthetized rats were transcardially perfused with $50 \mathrm{mM}$ phosphate-buffered saline (PBS), and fixed with a freshly prepared solution consisting of $4 \%$ paraformaldehyde (PFA) in $100 \mathrm{mM}$ phosphate buffer (PB) at $\mathrm{pH} 7.4$. Brains were dissected, post-fixed in the same fixative overnight, and transferred to $30 \%$ sucrose for cryoprotection. Coronal sections of $40 \mu \mathrm{m}$ thickness were made with a freezing microtome (Leica, Nussloch, Germany).

\section{TH immunohistochemistry}

For immunolabeling of $\mathrm{TH}$ in the striatum and $\mathrm{SN}, \mathrm{TH}$ immunohistochemistry was performed as the previously described method (Kim et al., 2011). Free-floating tissue sections were incubated overnight with mouse anti-TH antibody (1:1,000, Santa Cruz Biotechnology, Santa Cruz, CA, USA) and the sections were then incubated for $1 \mathrm{~h}$ with biotinylated anti-mouse secondary antibody (1:200, Vector Laboratories, Burlingame, CA, USA). The sections were subsequently incubated with avidin-biotin-peroxidase complex (Vector Laboratories) for $1 \mathrm{~h}$ at room temperature. Immunoreactivity was visualized by incubating the sections in a solution consisting of $0.05 \%$ 3,3-diaminobenzidine (DAB) and $0.01 \% \mathrm{H}_{2} \mathrm{O}_{2}$ in $50 \mathrm{mM}$ Tris-buffer ( $\mathrm{pH}$ 7.6) for approximately $3 \mathrm{~min}$. The sections were then washed three times with PBS 
and mounted onto gelatine-coated slides. The slides were air-dried overnight at room temperature, and coverslips were mounted using Permount ${ }^{\circledR}$.

\section{Data analysis}

TH-immunoreactive fiber density in the striatum was measured in $100 \mu \mathrm{m} \times 100 \mu \mathrm{m}$ square images of the striatum using animage analyzer (Multiscan, Fullerton, CA, USA). To estimate TH-staining density, optical densities were corrected for the nonspecific background density, which was measured in the completely denervated parts of the striatum. TH-positive fiber density ratios in the striatum were calculated as follows: optical density in the lesion side/optical density in the intact side. The number of TH-positive cells in the $\mathrm{SN}$ was counted hemilaterally through a light microscope (Olympus, Tokyo, Japan).

Statistical analysis was performed using one-way ANOVA followed by Duncan's post-hoc test, and the results are expressed as the mean \pm standard error of the mean (SEM). Significance was set as $P<0.05$.

\section{RESULTS}

\section{Effect of treadmill exercise on hyperactivity in the open field test}

The open field test was performed 29 days after starting of ex-

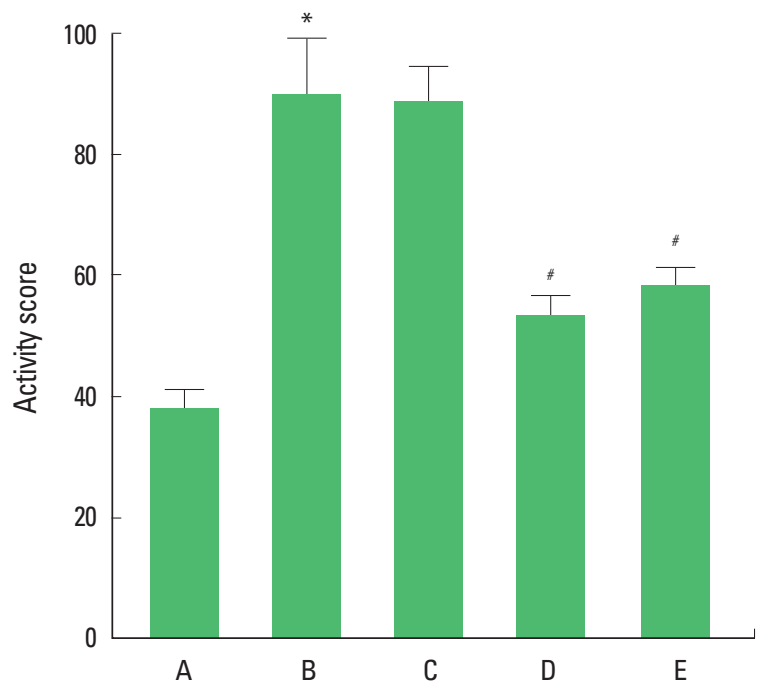

Fig. 1. Effect of treadmill exercise on hyperactivity in the open field test. (A) Control group. (B) attention deficit/hyperactivity disorder (ADHD) group. (C) $A D H D$ and treadmill exercise for 10 min group. (D) ADHD and treadmill exercise for 30 min group. (E) ADHD and treadmill exercise for 60 min group. *Represents $P<0.05$ compared to the control group. ${ }^{*}$ Represents $P<0.05$ compared to the ADHD group. periment. The activity score of the open field test are presented in Fig. 1 . The activity score was $38.20 \pm 2.73$ in the control group, $90.20 \pm 9.15$ in the ADHD group, $89.20 \pm 5.53$ in the $10 \mathrm{~min}$ treadmill exercise group, $53.60 \pm 3.31$ in the 30 min treadmill exercise group, and $58.60 \pm 2.99$ in the 60 min treadmill exercise group. These results show that the activity of in the ADHD rats was higher than that of the control rats. Treadmill exercise for 30 min most potently decreased the activity in the rats of the ADHD group.

\section{Effect of treadmill exercise on TH-immunoreactive fibers in the striatum}

Photomicrographs of the TH-immunoreactive fibers in the striatum are presented in Fig. 2. The optical density of the TH-immunoreactive fibers in the striatum was $127.18 \pm 2.96$ in the con-
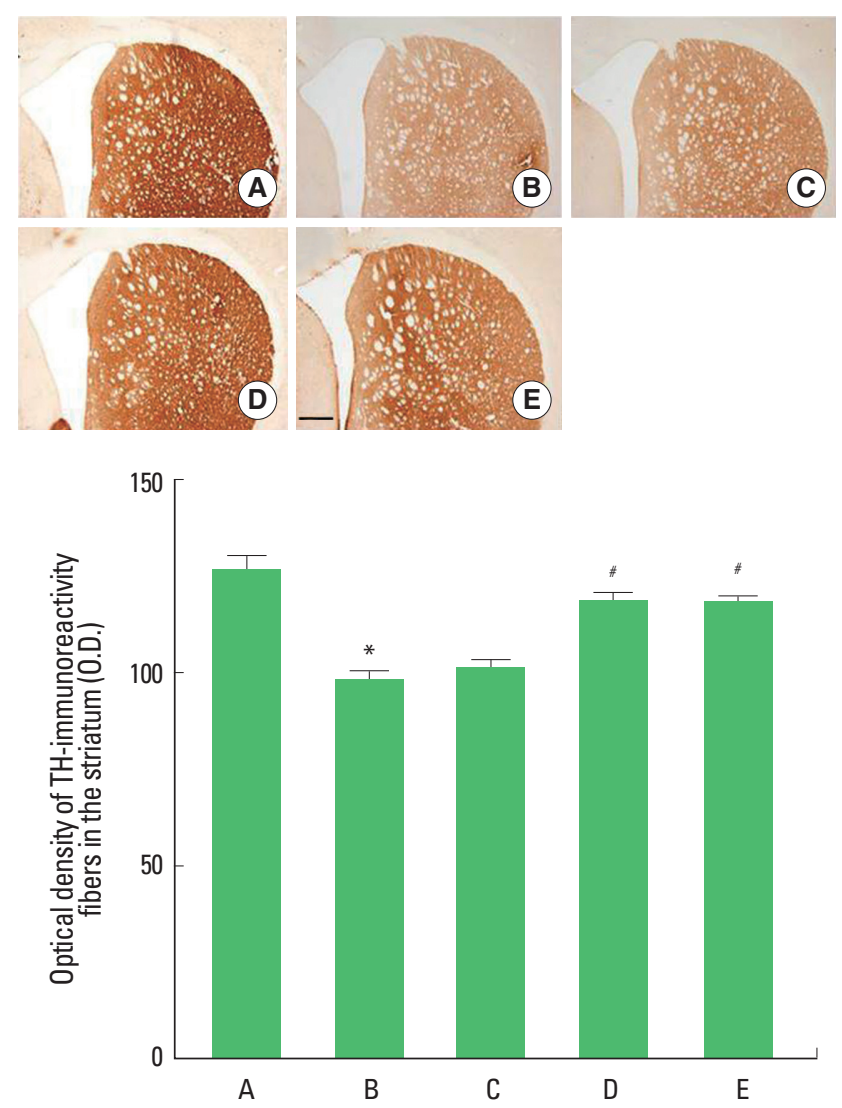

Fig. 2. Effect of treadmill exercise on tyrosine hydroxylase-immunoreactive fibers in the striatum. Upper: Photomicrographs of the tyrosine hydroxylase-positive fibers in the striatum. The scale bar represents $200 \mu \mathrm{m}$. (A) Control group. (B) attention deficit/hyperactivity disorder (ADHD) group. (C) ADHD and treadmill exercise for 10 min group. (D) ADHD and treadmill exercise for 30 min group. (E) ADHD and treadmill exercise for 60 min group. * Represents $P<0.05$ compared to the control group. "Represents $P<0.05$ compared to the ADHD group. 

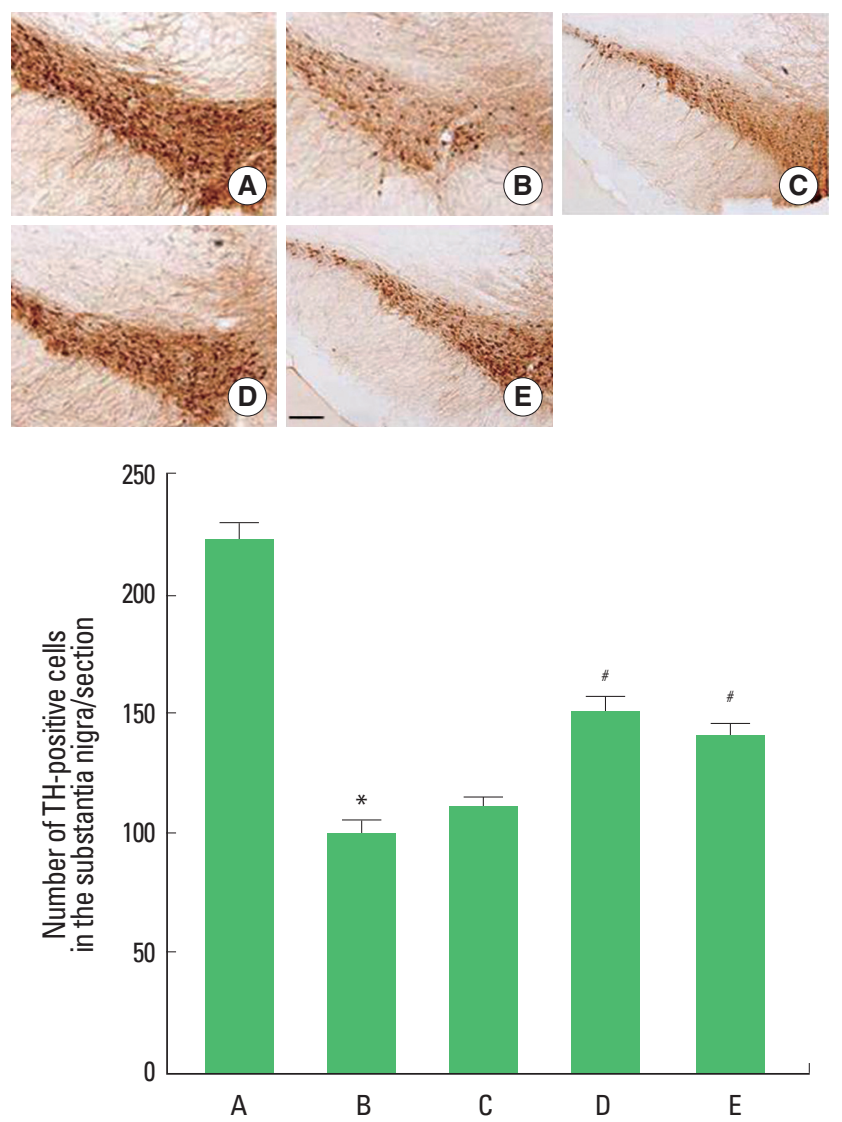

Fig. 3. Effect of treadmill exercise on tyrosine hydroxylase expression in the substantia nigra. Photomicrographs of tyrosine hydroxylase-positive cells in the substantial nigra. The scale bar represents $200 \mu \mathrm{m}$. (A) Control group. (B) Attention deficit/hyperactivity disorder (ADHD) group. (C) ADHD and treadmill exercise for 10 min group. (D) ADHD and treadmill exercise for 30 min group. (E) ADHD and treadmill exercise for 60 min group. *Represents $P<0.05$ compared to the control group. "Represents $P<0.05$ compared to the ADHD group.

trol group, $98.70 \pm 1.58$ in the ADHD group, $101.88 \pm 1.57$ in the $10 \mathrm{~min}$ treadmill exercise group, $119.06 \pm 1.60$ in the $30 \mathrm{~min}$ treadmill exercise group, and $118.53 \pm 0.97$ in the 60 min treadmill exercise group. The results show that $\mathrm{TH}$ fiber expression in the striatum was significantly decreased in the rats of the ADHD group than that of the control group. Treadmill exercise for 30 min most potently increased TH fiber expression in the rats of the ADHD group.

\section{Effect of treadmill exercise on TH expression in the SN}

Photomicrographs of the TH-positive cells in the $\mathrm{SN}$ are presented in Fig. 3. The number of TH-positive cells was 222.70 \pm 6.00 in the control group, $100.90 \pm 4.95$ in the ADHD group, $112.80 \pm 3.11$ in the 10 min treadmill exercise group, $151.20 \pm$ 6.11 in the $30 \mathrm{~min}$ treadmill exercise group, and $142.10 \pm 3.62$ in the 60 min treadmill exercise group. The results show that TH expression in the $\mathrm{SN}$ was significantly decreased in the rats of the ADHD group than that of the control group. Treadmill exercise for 30 min most potently increased TH expression in the rats of the ADHD group.

\section{DISCUSSION}

SHR is the most commonly accepted animal model for ADHD, because SHR strain shows hyperactivity (Sagvolden, 2000). This SHR displayed hyperactivity in the open field test and spatial learning memory deficit in the radial 8-arm maze task, as we expected. WKY rats, used as the control group, did not show any behavior symptoms of ADHD. The symptoms of ADHD, observed in the present study, are thought to be closely associated with hypo-function of dopaminergic system in some brain regions.

Dopamine is a neurotransmitter that is important in regulating brain processes involved with movement. Disease-induced loss of brain dopamine level leads to movement disorders (Servan-Schreiber et al., 1998). Dopamine is also important in regulating pleasurable responses as well as certain aspects of cognitive function, including attention (Sagvolden et al., 2005). These conditions give rise to delay aversion, development of hyperactivity in novel situations, impulsiveness, deficient sustained attention, increased behavioral variability (Sagvolden et al., 2005). Volkow et al. (2007) suggested that suppressed dopamine activity in caudate is associated with inattention in the adults with ADHD. Many studies have suggested the possibility that dysfunction of dopamine signaling in the midbrain is one of the main mechanisms of hyperactivity (Bowton et al., 2010).

In the present results, hyperactivity was observed in the ADHD rats, and expressions of $\mathrm{TH}$ in the striatum and $\mathrm{SN}$ were significantly decreased in the ADHD rats. The present study suggests that hyperactivity in the ADHD rats is associated with down-regulation of dopamine in the striatum and $\mathrm{SN}$.

Physical movement is known to improve working speed and social behavior problems and to diminishe hyperactivity in ADHD children (Majorek et al., 2004). Kim et al. (2011) reported that treadmill exercise showed alleviating effect on the hyperactivity in the ADHD rats. Increased dopamine synthesis by exercise enhanced the survival of dopaminergic neurons in the $\mathrm{SN}$ and treadmill running also alleviated some symptoms of ADHD (Hattori et al., 1994; Kim et al., 2011).

In the present results, treadmill exercise suppressed hyperactiv- 
ity and also inhibited $\mathrm{TH}$ expression in the striatum and $\mathrm{SN}$. Treadmill exercise for $30 \mathrm{~min}$ showed most potent inhibiting effect on hyperactivity, and this duration of treadmill exercise also most potently inhibited TH expression. Treadmill exercise for 10 min exerted no significant effects on activity and TH expression.

Here in this study, we showed that treadmill exercise can be considered therapeutic strategy for the symptom relief for ADHD. The present study suggests that treadmill exercise for 30 min once a day is the most effective therapeutic intervention for ADHD patients.

\section{CONFLICT OF INEREST}

No potential conflict of interest relevant to this article was reported.

\section{ACKNOWLEDGMENTS}

This study was supported by the Research Fund from Kyung Hee University granted in 2010 (KHU 20100648).

\section{REFERENCES}

Bedford TG, Tipton CM, Wilson NC, Oppliger RA, Gisolfi CV. Maximum oxygen consumption of rats and its changes with various experimental procedures. J Appl Physiol 1979;47:1278-1283.

Biederman J, Spencer T. Attention-deficit/hyperactivity disorder (ADHD) as a noradrenergic disorder. Biolo Psychiatry 1999; 46:1234-1242.

Bowton E, Saunders C, Erreger K, Sakrikar D, Matthies HJ, Sen N, Jessen T, Colbran RJ, Caron MG, Javitch JA, Blakely RD, Galli A. Dysregulation of dopamine transporters via dopamine D2 autoreceptors triggers anomalous dopamine efflux associated with attention-deficit hyperactivity disorder. J Neurosci 2010;30:6048-6057.

Durand M, Berton O, Aguerre S, Edno L, Combourieu I, Mormède P, Chaouloff $\mathrm{F}$. Effects of repeated fluoxetine on anxiety-related behaviours, central serotonergic systems, and the corticotropic axis axis in SHR and WKY rats. Neuropharmacology 1999;38:893-907.

Haavik J, Toska K. Tyrosine hydroxylase and Parkinson's disease. Mol Neurobiol 1998;16:285-309.

Hattori S, Naoi M, Nishino H. Striatal dopamine turnover during treadmill running in the rat: relation to the speed of running. Brain Res Bull 1994;35:41-49.

Heo YM, Shin MS, Lee JM, Kim CJ, Baek SB, Kim KH, Baek SS. Treadmill exercise ameliorates short-term memory disturbance in scopolamine-induced amnesia rats. Int Neurourol J 2014;18:16-22.
Hurley FM, Costello DJ, Sullivan AM. Neuroprotective effects of delayed administration of growth/differentiation factor-5 in the partial lesion model of Parkinson's disease. Exp Neurol 2004;185:281-289.

Jee YS, Ko IG, Sung YH, Lee JW, Kim YS, Kim SE, Kim BK, Seo JH, Shin MS, Lee HH, Cho HJ, Kim CJ. Effects of treadmill exercise on memory and c-Fos expression in the hippocampus of the rats with intracerebroventricular injection of streptozotocin. Neurosci Lett 2008;443:188192.

Kim BK, Shin MS, Kim CJ, Baek SB, Ko YC, Kim YP. Treadmill exercise improves short-term memory by enhancing neurogenesis in amyloid beta-induced Alzheimer disease rats. J Exerc Rehabil 2014;10:2-8.

Kim H, Heo HI, Kim DH, Ko IG, Lee SS, Kim SE, Kim BK, Kim TW, Ji ES, Kim JD, Shin MS, Choi YW, Kim CJ. Treadmill exercise and methylphenidate ameliorate symptoms of attention deficit/hyperactivity disorder through enhancing dopamine synthesis and brain-derived neurotrophic factor expression in spontaneous hypertensive rats. Neurosci Lett 2011;504:35-39.

Majorek M, Tüchelmann T, Heusser P. Therapeutic Eurythmy-movement therapy for children with attention deficit hyperactivity disorder (ADHD): a pilot study, Complement Ther Nurs Midwifery 2004;10: 46-53.

Nicola SM, Surmeier J, Malenka RC. Dopaminergic modulation of neuronal excitability in the striatum and nucleus accumbens. Annu Rev Neurosci 2000;23:185-215.

Pastor PN, Reuben CA. Diagnosed attention-deficit hyperactivity disorder and learning disability: United States, 2004-2006. Vital Health Stat 2008;10:1-14.

Sagvolden T. Behavioral validation of the spontaneously hypertensive rat (SHR) as an animal model of attention-deficit/hyperactivity disorder (AD/HD). Neurosci Biobehav Rev 2000;24:31-39.

Sagvolden T, Russell VA, Aase H, Johansen EB, Farshbaf M. Rodent models of attention-deficit/hyperactivity disorder. Biol Psychiatry 2005; 57:1239-1247.

Sagvolden T, Sergeant JA. Attention deficit/hyperactivity disorder from brain dysfunctions to behaviour. Behav Brain Res 1998;94:1-10.

Servan-Schreiber D, Bruno RM, Carter CS, Cohen JD. Dopamine and the mechanisms of cognition: Part I. A neural network model predicting dopamine effects on selective attention. Biol Psychiatry 1998;43:713722.

Volkow ND, Wang GJ, Newcorn J, Telang F, Solanto MV, Fowler JS, Logan J, Ma Y, Schulz K, Pradhan K, Wong C, Swanson JM. Depressed dopamine activity in caudate and preliminary evidence of limbic involvement in adults with attention-deficit/hyperactivity disorder. Arch Gen Psychiatry 2007;64:932-94.

Wilens TE, Dodson W. A clinical perspective of attention-deficit/hyperac- 
tivity disorder into adulthood. J Clin Psychiatry 2004;65:1301-1313.

Yoon MC, Shin MS, Kim TS, Kim BK, Ko IG, Sung YH, Kim SE, Lee HH,

Kim YP, Kim CJ. Treadmill exercise suppresses nigrostriatal dopami- nergic neuronal loss in 6-hydroxydopamine-induced Parkinson's rats. Neurosci Lett 2007;423:12-17. 